UCTP-105/97

\title{
Solution of gauge theories induced by fundamental representation scalars
}

\author{
P. Suranyi \\ University of Cincinnati, Cincinnati, Ohio 45221
}

\begin{abstract}
Gauge theories induced by scalars in the fundamental representation of the $U\left(N_{c}\right)_{\text {gauge }} \times U\left(N_{f}\right)_{\text {global }}$ group are investigated in the large $N_{c}$ and $N_{f}$ limit. A master field is defined from bilinears of the scalar field following an Eguchi-Kawai type reduction of spacetime. The density function for the master field satisfies an integral equation that can be solved exactly in two dimensions $(D=2)$ and in a convergent series of approximations at $D>2$. While at $D=2$ the system is in the same phase at all $\epsilon=N_{c} / N_{f}$, it undergoes a phase transition at a critical value, $\epsilon_{c}(D)$, for $D>2$.
\end{abstract}

\section{Introduction}

Many quantum field theories, with the notable exception of nonabelian gauge theories, can be solved exactly when the number of fields, $N$, tends to infinity. The large $N$ expansion is not overly useful for solving nonabelian gauge theories because the leading order of the expansion is equivalent to the sum of all planar diagrams. Unfortunately, it is not much simpler to sum planar diagrams than to solve the exact theory.

To obtain exact results further simplifications, like those proposed by Kazakov and Migdal [1], are required. Using the lattice action of a gauged adjoint representation scalar field, in which the pure gauge term was omitted, these authors derived an equation for the master field, composed of the eigenvalues of the scalar field matrix. Once the master field is known other physical parameters of the system can also be calculated. Following the philosophy that nonabelian gauge theories are unique one hopes that correct self-interactions of the gauge field are generated by scalar dynamics.

There are two reasons why gauge theories induced by adjoint representation scalars [1] [2] may not be completely equivalent to standard nonabelian theories. The first reason is well-known: Because the action is quadratic in the compact gauge field, $U$, there is a hidden local $Z_{N}$ symmetry, which implies that the vacuum expectation value of Wilson loops is zero. 33

The second reason which makes one believe that induced theories and standard theories are different, goes as follows. Suppose that along with Araf'eva [1] we gauge only one of the indices of the adjoint representation scalar field. Then the theory has a $U\left(N_{c}\right)_{\text {gauge }} \times U\left(N_{c}\right)_{\text {global }}$ symmetry and the unwanted $Z_{N}$ symmetry is not present. We can view such a theory as one having global and gauge symmetries with equal numbers of flavors and colors. The gauge theory induced by such a scalar field is not asymptotically free in four dimensions and as such is not likely to be confining either. In fact, the induced theory will only be asymptotically free if the number of 
complex scalars, $N_{f}$, satisfies the inequality $N_{f}>11 N_{c}$ [5], [6]. This is the primary reason why we previously considered a scalar field theory with $U\left(N_{c}\right)_{\text {gauge }} \times U\left(N_{f}\right)_{\text {global }}$ symmetry. [6]

The prevalent argument against theories with fundamental representation scalars, rather then adjoint representation ones has been the lack of existence of a master field, precluding chances for finding exact solutions. While in gauge theories induced by adjoint representation scalars the master field is composed of the eigenvalues of the scalar field matrix, for fundamental representation scalars no such quantity seems to exist.

The purpose of this paper is to show that the general bias against gauge theories induced by fundamental representation scalars is unfounded. It is possible to introduce an adjoint gauge tensor formed from bilinears of the fundamental field that can play a role identical to that of the primary scalar fields in previously investigated induced theories. A master field is formed from the eigenvalues of this positive definite self-adjoint matrix. The density function for the components of the master field satisfies a singular integral equation that can, in particular cases, be solved exactly or in a convergent series of approximations.

\section{Lattice model for gauged fundamental scalars}

Following the philosophy of induced gauge theories the Wilson plaquette term is omitted from a lattice action in the hope that correct gauge interactions are generated though scalar self-interactions. Then the only term of the Lagrangian in which the gauge field makes its appearance is the gauged kinetic term. The kinetic terms written in terms of fundamental representation scalars, $\psi_{\alpha}^{i}(x)$, and compact gauge fields, $U_{\mu}(x)$, has the form

$$
L_{K}=\kappa \sum_{\mu=1}^{D}\left[\psi_{i}^{\dagger}(x) U_{\mu}(x) \psi^{i}\left(x+a \hat{e}_{\mu}\right)+\psi_{i}^{\dagger}\left(x+a \hat{e}_{\mu}\right) U_{\mu}^{\dagger}(x) \psi^{i}(x)\right],
$$

where $\kappa$ is the hopping parameter, $a$ is the lattice constant. $i$ is a flavor index, while color indices are suppressed. $D$ is the number of dimensions and $\hat{e}_{\mu}$ is a unit vector along the $\mu$ th coordinate axis.

The hopping parameter can be eliminated by rescaling the scalar field and absorbing the change into the coefficients of the scalar potential. For that reason in what follows we will set $\kappa=1$.

The Lagrangian also contains interaction terms. We only introduce $\psi^{4}$ interaction terms but other terms relevant or marginal in less than four dimensions could also be introduced and treated without additional difficulty. In principle there are two fourth order self interaction terms allowed by the $U\left(N_{c}\right) \times U\left(N_{f}\right)$ symmetry, differing in their symmetry structure. These terms are formed from pairs of bilinears of the fundamental field that are global scalars and gauge tensors or scalars, or alternatively formed from gauge scalars that are global tensors or scalars. They have the form

$$
L_{I}=L_{T}+L_{S}=\frac{g_{T}}{N_{f}} \psi_{i}^{\dagger}(x) \psi^{j}(x) \psi_{j}^{\dagger}(x) \psi^{i}(x)+\frac{g_{S}}{N_{f}^{2}} \psi_{i}^{\dagger}(x) \psi^{i}(x) \psi_{j}^{\dagger}(x) \psi^{j}(x) .
$$

Terms with scalar coupling are not generated in the hopping parameter expansion. Thus, it is self-consistent to keep the term with the tensor structure only. Therefore, in what follows we will omit the scalar term and use the notation $g_{T}=g$.

The complete Lagrangian of the induced theory has then the form: $L=L_{K}+L_{T}+L_{M}$, where $L_{M}$ is the mass term,

$$
L_{M}=m^{2} \psi_{i}^{\dagger}(x) \psi^{i}(x)
$$


Following Araf'eva [4] we use an Eguchi-Kawai type realization [7] of coordinate space in the internal symmetry space. Such a reduction is admissible when the number of colors and flavors is large. In the large $N_{f}$ and $N_{c}$ limit one can realize the spacetime translation operators on the symmetry groups as $D_{\mu}(k)$ and $\Delta_{\mu}(p)$, where $D_{\mu}(k)$ is a diagonal $U\left(N_{c}\right)$ matrix, with matrix elements $e^{i a k_{\mu}^{\alpha}}, 1 \leq \alpha \leq N_{c}$, and $\Delta_{\mu}(p)$ is a diagonal $U\left(N_{f}\right)$ matrix, with matrix elements $e^{i a p_{\mu}^{i}}$, $1 \leq i \leq N_{f}$. These operators translate objects in the fundamental representation of the $S U\left(N_{c}\right)$ $\left[S U\left(N_{f}\right)\right]$ group by one lattice unit along the $\mu$ th axis. The model thus obtained called "quenched" because the integration over the momentum variables should be performed after physical amplitudes are calculated. Then the lagrangian is reduced to a single site form

$$
L=m^{2} \psi_{i}^{\dagger} \psi^{i}+\frac{g}{N_{f}} \psi_{i}^{\dagger} \psi^{j} \psi_{j}^{\dagger} \psi^{i}+\sum_{\mu} \operatorname{Tr}\left[\Delta_{\mu}(p) \psi_{i}^{\dagger} D_{\mu}^{1 / 2}(k) U_{\mu} D_{\mu}^{1 / 2}(k) \psi^{i}+h . c\right],
$$

where, for the sake of symmetry, we assume that the gauge fields occupy sites at the middle of links, halfway between two vertices of the lattice, where the scalar particles reside. It can be shown that the perturbation expansion of this model coincides with that of the original model at large $N_{f}$ and $N_{c}$.

At this point it can be easily seen that the translation operators can be eliminated from the theory altogether making the theory effectively translation invariant. The operator $\Delta_{\mu}(k)$ can be eliminated, because in gauge invariant closed loops its contributions cancel. The operators $D_{\mu}(k)$ can be eliminated using a redefinition of the gauge field. Then we arrive at the lagrangian

$$
L=m^{2} \psi_{i}^{\dagger} \psi^{i}+\frac{g}{N_{f}} \psi_{i}^{\dagger} \psi^{j} \psi_{j}^{\dagger} \psi^{i}+\sum_{\mu}\left[\psi_{i}^{\dagger} U_{\mu} \psi^{i}+h . c\right]
$$

which reproduces the correct perturbation expansion.

The theory defined by Lagrangian (5) fails in the weak coupling limit because it does not reproduce the correct eigenvalue spectrum of the matrices $U_{\mu}$. Therefore, one should introduce, along with Gross and Kitazawa [8] a quenched theory, containing a gauge invariant modification of the measure constraining the eigenvalues of $U_{\mu}$. The appropriately modified theory will be discussed briefly at the end of this paper and in more details in a future publication. Throughout this paper we will concentrate on the simple theory defined by Lagrangian (5).

At this point there is still no obvious candidate for a master field. Notice, however that lagrangian (5) depends only on the positive self adjoint tensor formed from bilinears of the scalar field, $\Phi$

$$
\Phi_{\alpha}^{\beta}=\psi_{\alpha}^{i} \psi_{i}^{\beta \dagger},
$$

where, for the sake of clarity the gauge indices $\alpha$ and $\beta$ are displayed.

We now change variables by introducing the following factor into the partition function

$$
1=\int d \Phi \delta\left(\Phi-\psi^{i} \psi_{i}^{\dagger}\right)=\int d \Phi d \rho e^{\operatorname{Tr} \rho\left(\Phi-\psi^{i} \psi_{i}^{\dagger}\right)} .
$$

Integrating over the complex scalar fields we obtain

$$
\int d \psi^{i}=\int d \Phi d \rho e^{\operatorname{Tr}\left[\rho \Phi-N_{f} \log \rho\right]} .
$$

At large $N_{f}$ we can evaluate the the integral over $\rho$ using the saddle point method. Thus, to terms of $O\left(1 / N_{f}\right)$ we obtain

$$
\int d \psi^{i} \sim \int d \Phi e^{N_{f} \operatorname{Tr} \log \Phi} .
$$


We are now in the position of writing down the complete action in terms of the fields $\Phi$ and $U$. Action $S$ will be defined with a sign opposite to the usual one so that $S=\log Z$, where $Z$ is the Boltzmann factor. We obtain

$$
S=\operatorname{Tr}\left[N_{f} \log \Phi-m^{2} \Phi-\frac{g}{N_{f}} \Phi^{2}+\sum_{\mu} \Phi\left(U_{\mu}+U_{\mu}^{\dagger}\right)\right] .
$$

The partition function is invariant under a unitary transformation of the positive hermitian matrix, $\Phi, \Phi \rightarrow V \Phi V^{\dagger}$, that diagonalizes $\Phi$. The action, $S$, written in terms of the eigenvalues of $\Phi, \phi_{\alpha}, \alpha=1, \ldots, N_{c}$ is

$$
S=\sum_{\alpha}\left[\frac{1}{2} \sum_{\beta \neq \alpha} \log \left(\phi_{\alpha}-\phi_{\beta}\right)^{2}+N_{f} \log \phi_{\alpha}-m^{2} \phi_{\alpha}-\frac{g}{N_{f}} \phi_{\alpha}^{2}\right]+D W(\phi),
$$

where

$$
W(\phi)=\log \int d U e^{\Phi\left(U+U^{\dagger}\right)}
$$

is the single link integral studied by Brower, Nauenberg, Brezin and Gross [9] [10] in the large $N$ limit, and $D$ is the number of dimensions. The first term of the action is the logarithm of the Jacobian of the transformation from $\Phi$ to its eigenvalues, $\phi_{\alpha}$.

As it will soon become clear the action is of $O\left(N_{f}^{2}\right)$. Then the most probable eigenvalues can be calculated by using the saddle point method. The values of $\phi_{\alpha}$ that maximize the action form the master field. By definition, all eigenvalues, $\phi_{\alpha}$, are positive. Zero eigenvalues are excluded by the term $\log \Phi$ of the action. At large $N$ these eigenvalues approximate a continuous distribution. As usual, we introduce the density of eigenvalues, $\rho(\nu)$, by the relation

$$
\frac{1}{N_{f}} \sum_{\alpha=1}^{N_{c}} \rightarrow \int d \nu \rho(\nu)
$$

where $\nu$ is the running eigenvalue. Consequently, $\rho(\nu)$ satisfies the sum rule

$$
\int \rho(\nu) d \nu=\epsilon=\frac{N_{c}}{N_{f}}
$$

The range of $\nu$ is yet unknown. The positivity of matrix $\Phi$ requires that it starts at some $\nu_{\min }>0$.

After rescaling variable $\nu$ by the relation $\nu \rightarrow \nu N_{f}, N_{f}$ can be eliminated and the action will have a nontrivial dependence on $\epsilon$ only. Using the results of [9] [10] and neglecting trivial constant terms we can write the action as

$$
\frac{S}{N_{f}^{2}}=\int d \nu \rho(\nu)\left[\frac{1}{2} \int d \lambda \rho(\lambda) \log (\nu-\lambda)^{2}+\log \nu-m^{2} \nu-g \nu^{2}\right]+D W
$$

where

$$
W=\int d \nu \rho(\nu)\left\{2\left(\nu^{2}+c\right)^{1 / 2}-\frac{1}{2} \int d \lambda \rho(\lambda) \log \left[\left(\nu^{2}+c\right)^{1 / 2}+\left(\lambda^{2}+c\right)^{1 / 2}\right]\right\}-c
$$

The constant $c$ is determined from the equation

$$
\int d \nu \frac{\rho(\nu)}{\left(\nu^{2}+c\right)^{1 / 2}}=2 .
$$


if condition

$$
s=\int d \nu \frac{\rho(\nu)}{\nu}>2 .
$$

is satisfied. This condition defines the strong coupling phase. If $s<2$ then $c=0$ and the system is in the weak coupling phase of the single site integral.

An integral equation for the optimal density distribution is obtained if one varies (15) with respect to the distribution function $\rho(\nu)$

$$
\int d \mu \rho(\mu)\left[\log (\mu-\nu)^{2}-D \log \left(\sqrt{\nu^{2}+c}+\sqrt{\mu^{2}+c}\right)\right]+\log \nu-m^{2} \nu+2 D \sqrt{\nu^{2}+c}-g \nu^{2}=\lambda,
$$

where $\lambda$ is a variational parameter, corresponding to constraint (14).

The derivative of (19) with respect to $\nu$ provides a singular integral equation that one hopes to solve for $\rho(x)$,

$$
P \int d \mu \rho(\mu)\left(\frac{2}{\nu-\mu}-\frac{\nu}{\sqrt{\nu^{2}+c}} \frac{D}{\nu+\mu}\right)+\frac{1}{\nu}-m^{2}+2 D \frac{\nu}{\sqrt{\nu^{2}+c}}-2 g \nu=0 .
$$

The solution of integral equation (20) does not determine the range of eigenvalues (variable $\nu$ ). In fact, one can get a solution for all choices of $0<\nu_{\min }<\nu_{\max }$. We need to impose an additional constraint, $\rho(\nu)>0$, to get a unique solution. The positivity of $\rho(\nu)$, as the density of eigenvalues is fairly obvious. Formally, it is required by the uniqueness of eigenvalues $\phi_{\alpha}$ of matrix $\Phi$ as a function of $\alpha$.

In principle we could obtain equations for $\nu_{\min }$ and $\nu_{\max }$ by varying the action with respect to these parameters. Being dependent on $d \nu \rho(\nu)$ only, these variations lead to (19), taken at the upper or lower limits and multiplied by the value of $\rho(\nu)$ taken at the same points. It follows then that the variation of the action has a double zero at a density $\rho(x)$ satisfying the variational equation and also vanishing at the end points. Consequently, the action has a point of inflection as a function of the end point parameters. If one slightly varies $\nu_{\min }$ (or $\nu_{\max }$ ) from the points at which $\rho\left(\nu_{\min }\right)=\rho\left(\nu_{\max }\right)=0$, with intention to increase $S$, then the density function becomes negative near the end point, leading to an inadmissible solution. Consequently, one must demand the vanishing of the density function $\rho(\nu)$ at the end points. As we will see later this condition determines the end points uniquely.

The method we use for determining the range of variation of eigenvalues can be applied to a wide range of problems involving matrix variables in the large $N$ limit and master fields. If the action is of the form

$$
S=\sum_{k} \prod_{i=1}^{k}\left[\int_{\nu_{\min }}^{\nu_{\max }} \mathrm{d} \nu_{i} \rho\left(\nu_{i}\right)\right] F_{k}\left(\nu_{1}, \ldots, \nu_{k}\right),
$$

then the spectral function must vanish at the boundaries, $\nu_{\min }$ and $\nu_{\max }$. In turn, this requirement can be used to determine $\nu_{\min }$ and $\nu_{\max }$.

We have not been able to find an exact solution of (20) at arbitrary $D$, either in the weak or in the strong coupling region. At $D=2$ an exact solution can be found in the weak coupling phase $(c=0)$. Furthermore, at $D>2$ the integral equation can be transformed into a Fredholm type equation providing a convergent expansion for $\rho(\nu)$, displaying critical behavior, around $D=2$ and a, probably continuous, phase transition as a function of $\epsilon$. The solution of the Fredholm equation at $D>2$ is also possible using a convergent expansion in a power series of $\epsilon$. 


\section{Exact solution of the model at $D=2$}

If the number of dimensions, $D=2$, then the problem of finding the density function simplifies considerably in the weak coupling phase of $W(a)$. To see this let us consider (20) in the weak coupling phase

$$
P \int d \mu \rho(\mu)\left[\frac{2}{\nu-\mu}-\frac{D}{\nu+\mu}\right]+\frac{1}{\nu}-M^{2}-2 \nu=0,
$$

where $M$ is the renormalized mass defined by $M^{2}=\frac{m^{2}-2 D}{\sqrt{g}}$. By redefining the mass parameter we scale out the coupling constant, $g$, from action. In the weak coupling phase it only appears in the rescaled relation $(17)$

$$
s=\sqrt{g} \int d \nu \frac{\rho(\nu)}{\nu}<2
$$

By an appropriate choice of $g$ it is always possible to tune $s$ to an arbitrary value inside the weak coupling phase, e.g to the boundary of the strong and weak coupling phases, where $s=2$. Note that in the strong coupling regime $c$ approaches zero continuously when the critical point $s=2$ is approached. Therefore, if $s$ is tuned to the critical point then our solution is valid, no matter which side the critical point is approached from.

At $D=2$ the derivative of variational equation (22) can be transformed to

$$
P \int_{-1}^{1} d x \frac{R(x)}{y-x}=-\frac{1}{\sqrt{A(1+z x)}}+M^{2}+2 \sqrt{A(1+z x)}
$$

where the constants $A, z$ and variable $x$ have been introduced by the relations

$$
\nu=\sqrt{A(1+z x)}, \quad \nu_{\max }=\sqrt{A(1+z)}, \quad \nu_{\min }=\sqrt{A(1-z)}, .
$$

and where we define $R(x)=\rho(\nu)$ In other words, the range of variable $x$ is $-1<x<1$.

Determining end points $\nu_{\max }$ and $\nu_{\min }$ is equivalent to determining $A$ and $z$. As we will see later these parameters are fixed by the requirement of $R(1)=R(-1)=0$.

A solution of integral equation (24) that vanishes at the end points, $x= \pm 1$, can be obtained by inverting the Cauchy principal value integral. After obvious manipulations one obtains the following expression for the density function

$$
\begin{aligned}
R(x) & =-\frac{\sqrt{1-x^{2}}}{4 \pi^{2}} P \int_{-1}^{1} \frac{\mathrm{d} y}{(x-y) \sqrt{1-y^{2}}}\left(-\frac{1}{\sqrt{A(1+z y)}}+M^{2}+2 \sqrt{A(1+z y)}\right) \\
& =\frac{\sqrt{A} z \sqrt{1-x^{2}}}{2 \pi^{2}} \int_{-1}^{1} \frac{2 \mathrm{~d} \xi}{(\sqrt{1+\xi z}+\sqrt{1+x z}) \sqrt{1-\xi^{2}}} \\
& +\frac{z \sqrt{1-x^{2}}}{4 \pi^{2} \sqrt{A} \sqrt{1+x z}} \int_{-1}^{1} \frac{\mathrm{d} \xi}{(\sqrt{1+\xi z}+\sqrt{1+x z}) \sqrt{1+\xi z} \sqrt{1-\xi^{2}}}
\end{aligned}
$$

The density, $R(x)$, is obviously positive definite and as such, physically admissible. We could express $R(x)$ in a closed form as a combination of complete elliptic functions. As it is not especially illuminating that form will not be presented in this paper.

At this point $A$ and $z$ are yet undetermined. It is comparatively easy to see that consistency conditions allow us to determine these constants. Before discussing these conditions it is worth mentioning that solutions of integral equation (24), not vanishing at $x= \pm 1$, could be found at 
every choice of the end points. These solutions would either not maximize $S$ or would not be positive definite.

To determine the end points $\nu_{\min }$ and $\nu_{\max }$ (i.e. parameters $A$ and $z$ ) first note that form (26) seems to be independent of $M^{2}$. This is so because when the Cauchy integral is inverted the term $M^{2}$ on the right hand side of (26) gives a vanishing contribution. This term can however be recovered if we substitute expression (26) into (24) and use the Poincare-Bertrand formula for the exchange of the order of Cauchy integrals. Then all terms of the right hand side of (24) except $M^{2}$ are recovered. A constant term appears that must be identified with $M^{2}$ :

$$
\frac{1}{\pi} \int_{-1}^{1} \frac{\mathrm{d} \xi}{\sqrt{1-\xi^{2}}}\left(\frac{1}{\sqrt{A(1+z \xi)}}-2 \sqrt{A(1+z \xi)}\right)=M^{2} .
$$

This equation can be used to determine $A$ in terms of $z$ and $M$.

The second condition is obtained from the normalization of the spectral function, (14). After simple manipulations we obtain the following relation:

$$
-\frac{1}{2 \pi} \int_{-1}^{1} \frac{\xi \mathrm{d} \xi}{\sqrt{1-\xi^{2}}}\left(\frac{1}{\sqrt{A(1+z \xi)}}-2 \sqrt{A(1+z \xi)}\right)=\epsilon
$$

Having determined $A$ as a function of $z$ and $M$ from (27) we can use (28) to determine $z$ as a function of the physical parameters $M$ and $\epsilon$.

Numerical calculations show that one can find unique positive solutions for $A$ and $z$ at all possible choices of real $M^{2}$ and positive $\epsilon$. As a contrast, we will see later at $D>2$ we can find a similar type of solution only in a restricted range of $\epsilon . D=2$ is a lower critical dimension of the theory.

We now examine the behavior of the solution in the limits of small and large $\epsilon$. At small values of $\epsilon=N_{c} / N_{f} z \sim \epsilon$. In other words, the range of eigenvalues relative to the average value shrinks to zero. Then the matrix $\Phi$ tends to a constant multiple of the unit matrix. We can also see by inspection that in the limit $z \rightarrow 0$ the distribution of eigenvalues follows Wigner's semicircle law.

At large $\epsilon \quad z \sim 1-e^{-\epsilon^{2}}$, the lower bound of the eigenvalues of matrix $\Phi, \nu_{\min }=A(1-z) \sim$ $\epsilon e^{-\epsilon^{2}}$, approaches zero, while the upper limit, $\nu_{\max }=A(1+z) \sim 2 \epsilon$, tends to infinity, so that the eigenvalues span the whole positive real axis. The distribution of eigenvalues, $R(x)$, differs substantially from the semicircle law, favoring eigenvalues close to the lower bound.

\section{Induced theories at $D>2$}

At $D>2$ we have not been able to find an exact solution for the density function. It turns out however that even at $D>2$ the singular integral equation can be transformed into a Fredholm equation, solutions of which can be generated in convergent expansions. Having these expansions is almost as useful as having an exact solution.

We will start again from the derivative of (22) with respect to $\nu$. Using variable $x$ and parameters $A$ and $z$ as at $D=2$, in (24) we obtain

$$
\begin{aligned}
P \int_{-1}^{1} d x \frac{R(x)}{y-x} & =\frac{(D-2) z}{2} \int_{-1}^{1} \frac{R(x) \mathrm{d} x}{\sqrt{1+z x}(\sqrt{1+z x}+\sqrt{1+z y})} \\
& -\frac{1}{\sqrt{A(1+z x)}}+M^{2}+2 \sqrt{A(1+z x)}
\end{aligned}
$$


This equation is identical to (24) other then the appearance of the first term on the right hand side.

After inverting the Cauchy integral on the left hand side of (29) we obtain

$$
\begin{aligned}
R(x) & =f(x)+(D-2) \int_{-1}^{1} \mathrm{~d} y K(x, y) R(y) \\
& \equiv \frac{\sqrt{A} z \sqrt{1-x^{2}}}{2 \pi^{2}} \int_{-1}^{1} \frac{2 \mathrm{~d} \xi}{(\sqrt{1+\xi z}+\sqrt{1+x z}) \sqrt{1-\xi^{2}}} \\
& +\frac{z \sqrt{1-x^{2}}}{4 \pi^{2} \sqrt{A} \sqrt{1+x z}} \int_{-1}^{1} \frac{\mathrm{d} \xi}{(\sqrt{1+\xi z}+\sqrt{1+x z}) \sqrt{1+\xi z} \sqrt{1-\xi^{2}}} \\
& -\frac{(D-2) z^{2} \sqrt{1-x^{2}}}{4 \pi^{2}} \int_{-1}^{1} \frac{R(y) \mathrm{d} y}{(\sqrt{1+y z}+\sqrt{1+x z}) \sqrt{1+y z}} \\
& \times \int_{-1}^{1} \frac{\mathrm{d} \xi}{(\sqrt{1+\xi z}+\sqrt{1+x z})(\sqrt{1+\xi z}+\sqrt{1+y z}) \sqrt{1-\xi^{2}}}
\end{aligned}
$$

This is a linear, inhomogeneous integral equation with an inhomogeneity that is identical to the density function at $D=2$, with kernel

$$
\begin{aligned}
K(x, y) & =-\frac{z^{2} \sqrt{1-x^{2}}}{4 \pi^{2}(\sqrt{1+y z}+\sqrt{1+x z}) \sqrt{1+y z}} \\
& \times \int_{-1}^{1} \frac{\mathrm{d} \xi}{(\sqrt{1+\xi z}+\sqrt{1+x z})(\sqrt{1+\xi z}+\sqrt{1+y z}) \sqrt{1-\xi^{2}}}
\end{aligned}
$$

and with expansion parameter $D-2$. Kernel $K(x, y)$ can easily be symmetrized. It is bounded, i.e. it satisfies condition

$$
\int_{-1}^{1} \mathrm{~d} x \int_{-1}^{1} \mathrm{~d} y|K(x, y)|^{2}<\infty
$$

It follows that (30) is a Fredholm equation.

The solution of the integral equation is given by the following expression

$$
R(x)=f(x)+\int_{-1}^{1} \mathrm{~d} y \operatorname{Res}(x, y) f(y),
$$

where $f(x)$ is the inhomogeneity of the integral equation, and $\operatorname{Res}(x, y)$ is the resolvent of the equation,

$$
\operatorname{Res}(x, y)=\frac{(D-2) K(x, y)}{1-(D-2) K(x, y)}=\frac{N(x, y)}{\operatorname{Det}} .
$$

The Fredholm numerator, $N(x, y)$, and the Fredholm determinant, Det, are entire functions of the expansion parameter, $D-2$. The first terms of their expansions are given by

$$
\begin{aligned}
N(x, y) & =(D-2) K(x, y)-\frac{(D-2)^{2}}{2 !}\left(K(x, y) \operatorname{Tr} K-K^{2}(x, y)\right)+\ldots \\
\text { Det } & =1-(D-2) \operatorname{Tr} K+\frac{(D-2)^{2}}{2 !}\left[(\operatorname{Tr} K)^{2}-\operatorname{Tr} K^{2}\right]
\end{aligned}
$$

where $K^{2}$ is understood in the operator sense. 
The negative definite kernel insures that at $D>2$ the Fredholm determinant has no zeros. At zeros of the Fredholm determinant only the homogeneous equation has a solution. Thus, the only reason why solutions of (30) would not be acceptable is the lack of positivity at all $x$.

Note that at $z=0$ the kernel vanishes. Then, in view of the positivity of the inhomogeneity at all $-1<x<1$ the spectral function is physically acceptable. Since small $z$ and small $\epsilon$ are synonymous, we find that at all $D>2$ one can find a critical value of $\epsilon$, such that for $\epsilon<\epsilon_{c}(D)$ a physically acceptable solution exists. As we saw earlier $\epsilon_{c}(2)=\infty$.

By examining the solution at $D=2$ one can see that $z \rightarrow 1$ corresponds to $\epsilon \rightarrow \infty$. Therefore, one would expect that at $D>2$ limitations on the range of admissible values of $z$ may appear near $z=1$ (large $\epsilon$ ), excluding large values of $\epsilon$. Therefore we performed a systematic expansion of the Fredholm determinant and solution $R(x)$ near $z=1$.

Due to singularities at $x=-1$ the terms of Det (and of $N(x, y)$ ) diverge logarithmically near $z=1$. The Fredholm determinant can be written as

$$
\text { Det }=\exp \{\operatorname{Tr} \log (1-(D-2) K)\}=\exp \left\{-(D-2) \operatorname{Tr} K-\frac{(D-2)^{2}}{2} \operatorname{Tr} K^{2}+\ldots\right\} .
$$

It is easy to calculate the leading (and even subleading) logarithms of the traces of powers of the kernel. Using these we obtain the following expression for the Fredholm determinant near $z=1$ :

$$
\text { Det }=(1-z)^{\alpha(D)},
$$

where

$$
\alpha(D)=\frac{1}{\pi^{2}}\left[\frac{D-2}{2}-\frac{(D-2)^{2}}{3}+O\left((D-3)^{3}\right)\right] .
$$

This is a critical exponent. Due to the rearrangement of the Fredholm series, this expansion, as similar perturbation expansions, is asymptotic only.

The relation of parameters $A$ and $z$ to $M^{2}$ and $\epsilon$ is similar to relations (27) and (28):

$$
\begin{aligned}
& \frac{1}{\pi} \int_{-1}^{1} \frac{\mathrm{d} \xi}{\sqrt{1-\xi^{2}}}\left(\frac{1}{\sqrt{A(1+z \xi)}}-2 \sqrt{A(1+z \xi)}\right. \\
- & \left.\frac{(D-2) z}{2} \int_{-1}^{1} \frac{R(y) \mathrm{d} y}{\sqrt{1+z y}(\sqrt{1+z \xi}+\sqrt{1+z y})}\right)=M^{2} .
\end{aligned}
$$

and

$$
\begin{aligned}
& -\frac{1}{2 \pi} \int_{-1}^{1} \frac{\xi \mathrm{d} \xi}{\sqrt{1-\xi^{2}}}\left(\frac{1}{\sqrt{A(1+z \xi)}}-2 \sqrt{A(1+z \xi)}\right. \\
& \left.-\frac{(D-2) z}{2} \int_{-1}^{1} \frac{R(y) \mathrm{d} y}{\sqrt{1+z y}(\sqrt{1+z \xi}+\sqrt{1+z y})}\right)=\epsilon .
\end{aligned}
$$

We will investigate now the positivity of the density function. It is fairly easy to show that in the immediate neighborhood of $x=-1$, where the kernel and inhomogeneity become singular at $z=1$, the iterated density function is positive definite. Therefore we will concentrate on the region of $x+1>>1-z$.

The first nontrivial approximation to the Fredholm series is identical to the once iterated solution of the integral equation. It provides an asymptotic relation for the range of $\epsilon$. To see this we investigate the dependence of $A$ on $z$. This must be done before one is able to calculate the leading 
contributions to the density function. If we study solutions at vanishing or small renormalized mass. $M$, then the location of the critical point is independent of $M$ in leading orders of the expansion.

We calculated nonleading order corrections, as well, just to see how much they influence asymptotic relationships. Using (40) we obtain

$$
A=\log \left(\frac{2}{1-z}\right) \frac{1-(D-2) \log [2 /(1-z)] / 192 \pi^{2}}{8+0.82247(D-2) / \pi^{2}}
$$

When the leading asymptotic part of this relation is substituted into the expression of the density function we obtain

$$
R(x) \simeq \frac{\sqrt{1-x^{2}}}{(1+z x) 2 \sqrt{2} \pi^{2} \sqrt{A}} \log \left(\frac{2}{1-z}\right)\left(1-\frac{(D-2) \log ^{2}\left(\frac{2}{1-z}\right)}{12 \sqrt{2} \pi^{2}}\right) .
$$

The density function becomes indefinite at large enough $D-2$. This is a nonsingular structural phase transition, except in the vicinity of $D=2$. Near the transition $(D-2)(\log [2 /(1-z)])^{2}=$ $O(1)$, so at large $\log [2 /(1-z)]$ the corrections in (42) are indeed small.

Having determined the critical value of $z$ (the maximal $z$ with admissible spectral function) we can determine the critical value of $\epsilon$ using (41). Substituting into this relation we get

$$
\epsilon=\sqrt{\log \left[\frac{2}{1-z}\right]} \frac{4}{3 \pi}+\ldots
$$

Combining (44) and (43) we get the final expression for the critical value of $\epsilon$

$$
\epsilon_{c}=\frac{1}{(D-2)^{1 / 4}}\left(\frac{1024 \sqrt{2}}{27 \pi^{2}}\right)^{1 / 4}=\frac{2.331}{(D-2)^{1 / 4}} .
$$

As expected, at $D=2$ the critical value of $\epsilon$ diverges. The prediction of (45) for the value of $\epsilon_{c}(D)$ is not reliable at $D=4$. A better value could be obtained from a thorough numerical study.

It is interesting to speculate what happens at $\epsilon>\epsilon_{c}(D)$. It is easy to see that density functions, satisfying $\rho(\nu)>0$ and having finite action still exist. The positive density function that maximizes $S$ at $\epsilon>\epsilon_{c}$ may not belong to the class of density functions we have been investigating above. One is able to get a hint about the nature of the density function in this region if one considers that when $\epsilon \rightarrow \epsilon_{c}(D)-0$ then $R(x)$ develops a zero at some intermediate value of $x, x=x_{c}$, where $-1<x_{c}<1$. Then at $\epsilon>\epsilon_{c}(D)$ the spectrum of eigenvalues should split into two disjunct intervals, the density function vanishing at the end points of both intervals. This would be a density function that does not belong to the class of functions considered above. Clearly, the change from one to the other type of density functions is, an almost probably continuous, structural phase transition.

To study the nature of these phases it would be very important to derive the dependence of large Wilson loops,

$$
W[X, Y]=\left\langle\operatorname{Tr}\left[\left(U_{x}\right)^{X}\left(U_{y}\right)^{Y}\left(U_{x}\right)^{-X}\left(U_{y}\right)^{-Y}\right]\right\rangle .
$$

on the area of the loop, $X Y$. This, and the investigation of the model in the strong coupling regime of the single link integral are left to a future publication. 


\section{Quenched Theory}

In the above described theory, in the weak coupling phase, the distribution of eigenvalues of the gauge matrices is incorrect. To eliminate this problem one must constrain the eigenvalues of the gauge field $U_{\mu}$ in a gauge invariant manner. One possible approach is that of Gross and Kitazawa [8] that introduces the following modification of the measure:

$$
\int d U_{\mu} \rightarrow \int d U_{\mu} \prod_{\mu} \int d V_{\mu} \Delta\left(D_{\mu}\right) \delta\left(D_{\mu}^{1 / 2} U_{\mu} D_{\mu}^{1 / 2}-V_{\mu} D_{\mu} V_{\mu}^{\dagger}\right) .
$$

While this addition to the measure fixes the eigenvalue distribution of matrices $U_{\mu}$, the substitution does not affect agreement with the perturbation expansion of the original theory.

Substituting into the Lagrangian we obtain

$$
L=m^{2} \psi_{i}^{\dagger} \psi^{i}+\frac{g}{N_{f}} \psi_{i}^{\dagger} \psi^{j} \psi_{j}^{\dagger} \psi^{i}+\kappa \sum_{\mu}\left[\psi_{i}^{\dagger} V_{\mu}\left(D_{\mu}+D_{\mu}^{\dagger}\right) V_{\mu}^{\dagger} \psi^{i}\right]
$$

At this point one can introduce the bilinear combinations just as in (6) to get the quenched theory for the positive adjoint matrix $\Phi$, The action, in terms of the eigenvalues of $\Phi, \phi_{\alpha}$ takes the form

$$
S=\sum_{\alpha}\left[\frac{1}{2} \sum_{\beta \neq \alpha} \log \left(\phi_{\alpha}-\phi_{\beta}\right)^{2}+N_{f} \log \phi_{\alpha}-m^{2} \phi_{\alpha}-\frac{g}{N_{f}} \phi_{\alpha}^{2}\right]+\sum_{\mu} \log I\left(\Phi, C_{\mu}\right),
$$

where $I\left(\Phi, C_{\mu}\right)$ is the Itzykson-Zuber integral [1]

$$
I\left(\Phi, C_{\mu}\right)=\frac{\operatorname{Det}\left[e^{2 \kappa \phi_{\alpha} \cos k_{\mu}^{\beta}}\right]}{\Delta\left(\phi_{\alpha}\right) \Delta\left(\cos k_{\mu}^{\beta}\right)}=\int d V_{\mu} e^{2 \kappa \sum_{\mu} \operatorname{Tr} \Phi V_{\mu} C_{\mu} V_{\mu}^{\dagger}}
$$

and where

$$
\Delta\left(a_{\alpha}\right)=\prod_{\alpha \neq \beta}\left(a_{\alpha}-a_{\beta}\right)
$$

and $C_{\mu}$ is a diagonal matrix formed from the cosines of the momentum variables.

The density function for the distribution of continuous eigenvalues can proceed the same way as it was done in the previous sections. The singular integral equation derived from the variation of the action is complicated but can be studied in the weak and strong coupling limits of the Itzykson-Zuber integral.

Finally, we present the expression of Wilson loops in terms of the variables used in this section: The Wilson loop of edges $X$ and $Y$ along the $x$ and $y$ axes is defined in the quenched theory as

$$
\begin{aligned}
\langle W(X, Y)\rangle & =\operatorname{Tr}\left[\left(D_{x}^{1 / 2} U_{x} D_{x}^{1 / 2}\right)^{X}\left(D_{y}^{1 / 2} U_{\nu} D_{y}^{1 / 2}\right)^{Y}\left(D_{x}^{1 / 2} U_{x} D_{x}^{1 / 2}\right)^{-X}\left(D_{y}^{1 / 2} U_{\nu} D_{y}^{1 / 2}\right)^{-Y}\right] \\
& =\operatorname{Tr}\left[V_{x} e^{i X k_{x}} V_{x}^{\dagger} V_{y} e^{i Y k_{y}} V_{y}^{\dagger} V_{x} e^{-i X k_{x}} V_{x}^{\dagger} V_{y} e^{-i Y k_{y}} V_{y}^{\dagger}\right]
\end{aligned}
$$

\section{Conclusion}

The investigation of gauge theories induced by scalars in the fundamental representation may solve some of the problems that have arisen in theories induced by adjoint representation scalars. 
Prominent among these is the vanishing of the expectation value of the Wilson loop. This is the main reasons why we started an investigation of these theories.

The most serious difficulty in solving theories induced by fundamental representation scalars has been the apparent lack of candidates for a master field. We solved this problems by showing that bilinears formed from fundamental representation fields form self adjoint matrices the eigenvalues of which are the perfect candidates for a master field.

Using an Eguchi-Kawai type reduced representation we obtained an integral equation for the density of eigenvalues that could be solved exactly in two dimensions at all values of the physical parameters. At $D>2$ we obtained a Fredholm integral equation for the density function. Using the perturbative solution of this equation we established that the spectrum undergoes a phase transition at a critical value of $\epsilon=N_{f} / N_{c}$. Further investigations are needed to examine the nature of the phase transition at $D>2$ and the size dependence of the expectation value of Wilson loops.

The translation invariant approximation used in most of this paper should be complemented by a non-translation invariant approach. Investigation of theories induced by fundamental scalars using the Gross-Kitazawa quenching prescription [8] is the next step of investigations.

\section{Acknowledgment}

This work was supported in part by the U.S. Department of Energy Grant \#DE-FG02-84ER40153.

\section{References}

[1] V. A. Kazakov and A. A. Migdal, Nucl. Phys. B397 (1993) 214

[2] D. J. Gross, Phys. Lett. B293 (1992) 181;

[3] I. I. Kogan, G. W. Semenoff, and N. Weiss, Semenoff, Phys. Rev. Lett. 69 (1992) 3435;

[4] I. Ya. Araf'eva, Phys. Lett. B308 (1993) 347

[5] A. Hasenfratz and P. Hasenfratz, Phys. Lett. B297 (1992) 166;

[6] A. Pap and P. Suranyi, Phys. Rev. D55 (1997) 3743;

[7] T. Eguchi and H. Kawai, Phys. Rev. Lett. 48 (1982) 1063;

[8] D. J. Gross and Y. Kitazawa, Nucl. Phys. B206 (1982) 440;

[9] R. Brower and M. Nauenberg, Nucl. Phys. B180 (FS2) (1981) 221;

[10] E. Brezin and D. J. Gross, Phys. Lett. 97B (1980) 120

[11] C. Itzykson and J. B. Zuber, Journ. Math. Phys. 21 (1980) 411. 